\title{
Protective effect of hydrogen sulfide is mediated by negative regulation of epigenetic histone acetylation in Parkinson's disease
}

\author{
Yang Sun ${ }^{1}$, Dai Li ${ }^{2}$, Yuqiang Su ${ }^{3}$, Haikang Zhao ${ }^{4}$, Weiwei Pang ${ }^{1}$, Wei Zhao ${ }^{1}$, Shengjun $\mathrm{Wu}^{5}$
}

${ }^{1}$ Department of Rehabilitation Medicine, The Second Affiliated Hospital of Xi'an Medical University, Xi'an, Shaanxi, China

2Department of Anesthesiology, Chang Hai Hospital, Naval Military Medical University, Shanghai, China

${ }^{3}$ Department of Anesthesiology, The Second Affiliated Hospital of Xi'an Medical University, Xi'an, Shaanxi, China

${ }^{4}$ Department of Neurosurgery, The Second Affiliated Hospital of Xi'an Medical University, Xi'an, Shaanxi, China

${ }^{5}$ Department of Gynecology, The Second Affiliated Hospital of Xi'an Medical

University, Xi'an, Shaanxi, China

Submitted: 13 June 2019

Accepted: 14 October 2019

Arch Med Sci

DOI: https://doi.org/10.5114/aoms.2020.93121

Copyright $\odot 2020$ Termedia \& Banach

\section{Abstract}

Introduction: Derangements in monoaminergic transmission in the substantia nigra with disturbed signaling in the hypothalamic-pituitary-adrenal axis are the major characteristics of Parkinson's disease (PD). It has been reported that the administration of hydrogen sulfide $\left(\mathrm{H}_{2} \mathrm{~S}\right)$ is in practice to treat PD because of its redundant nature in regulating various neuronal signals. Hence, the current investigation was performed to evaluate the hypothesis that $\mathrm{H}_{2} \mathrm{~S}$ might exert protective action via the inhibition of epigenetic histone acetylation.

Material and methods: To test this notion, 6-hydroxydopamine (6-OHDA) was used to induce PD and sodium hydrogen sulfide (SHS) was used as a $\mathrm{H}_{2} \mathrm{~S}$ donor and tubastatin A (TSA) was tested in an in vivo rat model to delineate the signaling mechanism.

Results: Induction of PD in rats demonstrated elevated oxidative stress with an evidenced decrease in antioxidant enzymes, while elevated pro-inflammatory cytokines and inflammatory mediators were observed in the striatum of PD rats compared to controls. On the other hand, elevated $(p<0.01)$ levels of dopamine, 3,4-dihydroxyphenylacetic acid (DOPAC), mRNA transcript of HDAC-2, $-3,-4,-6$ and total histone deacetylase (HDAC) were found with reduced levels of histone acetyltransferase (HAT) in the brain tissues of PD induced rats.

Conclusions: Diversely, $\mathrm{H}_{2} \mathrm{~S}$ exposure reversed these alterations with reduced HDAC activity. Further, PD rats treated with HDAC inhibitor showed a dramatic upsurge in the level of tyrosine hydroxylase, with a decreased level of glial fibrillary acidic protein, $\alpha$-synuclein, tumor necrosis factor $\alpha$, and other cytokines. Thus the results of the study suggest that $\mathrm{H}_{2} \mathrm{~S}$ exerts protection via inhibition of HDAC.

Key words: Parkinson's disease, hydrogen sulfide, histone deacetylase, histone acetyltransferase, cytokines.

\author{
Corresponding author: \\ Shengjun Wu \\ Department \\ of Gynecology \\ The Second Affiliated \\ Hospital of Xi'an \\ Medical University \\ Xi'an, Shaanxi, China \\ E-mail: \\ ColtPittspyg@yahoo.com
}




\section{Introduction}

The age-linked neurodegenerative Parkinson's disease (PD) is the main reason for disability of ageing worldwide. It imposes a burden on the socio-economic welfare of the society. PD is considered to involve advanced degeneration of dopaminergic neurons (DN) in the substantia nigra of the midbrain and depletion of the neurotransmitter dopamine (DA) in the striatum [1, 2]. The symptoms are debilitating and include slow movement, rigidity, resting tremor, bradykinesia and postural inability [3]. Lewy bodies that are the cytoplasmic aggregate of $\alpha$-synuclein protein along with the loss of DN are the distinctive features of the disease. The primary cause of nigral dopaminergic cell death in PD is oxidative injury and a neurotoxin that causes oxidative stress by binding to the dopamine transporter and delivers its toxicity to DN by generating reactive oxygen species (ROS) such as superoxide radicals [4-6] and kills them.

Treatment of PD involves usage of the drug levodopa, which removes the DA deficiency. But prolonged treatment with levodopa has long term side effects that include dyskinesia and it does not arrest the neurodegeneration. Recently, hydrogen sulfide, which is a toxic and flammable gas, has been used to treat PD. $\mathrm{H}_{2} \mathrm{~S}$ that is produced by the enzymatic actions of cystathionine b-synthase and cystathionine c-lyase on L-cysteine would act to regulate cell proliferation, apoptosis, regulation of insulin release, calcium homeostasis and control of oxidative stress [7-13]. The administration of $\mathrm{H}_{2} \mathrm{~S}$ in combating oxidative stress and to give neuroprotection has already been reported for neurodegenerative diseases such as Alzheimer's and vascular dementia [14-16]. Intracytoplasmic protein inclusions known as Lewy bodies are primarily composed of synaptic protein called $\alpha$-synuclein that accumulates in the substantia nigra pars compacta (SNpc) of DN due to degeneration of dopaminergic nigrostriatal pathways $[17,18]$ and are primarily responsible for the motor-related symptoms in PD. Furthermore, activated microglia activates the brain's innate immune response, which results in the secretion of pro-inflammatory cytokines, such as tumor necrosis factor- $\alpha$ (TNF- $\alpha$ ), interleukin-6 (IL-6) and interleukin-1 $\beta$ [19].

The pathogenicity of PD has been associated with the epigenetics where histone modifications and DNA methylations could hold the key. Histone deacetylase (HDAC) enzymes mediate the deacetylation of $\mathrm{N}$-terminal lysine residues of core histones. It affects the chromatin structure and condenses it, thereby preventing access to transcribe certain genes that are important in regulating the neurological and inflammatory pathologies. Histone acetyl transferases (HAT) attempt to reverse the activities of HDAC. The activities of HDAC and HAT are important in maintaining the cell homeostasis in which the gene transcription is in dynamic equilibrium between the two $[20,21]$ and any imbalance seen in these cases seems to be the hallmark of onset of the disease. Various researchers have demonstrated such premonitions in the development of neurodegeneration in Alzheimer's disease, lateral sclerosis [22] and in experimental models of PD [23]. HDAC are natural targets and inhibiting their activity has been reported to rescue the cells which are leading to cell death in the associated pathways in PD, giving valuable leads in the treatment of this disease.

With this observation on the possible notes of HDAC, the present study was performed using 6-hydroxydopamine (6-OHDA), a neurotoxin that is used widely [24-26] to induce PD in animal models and the impact of HDAC inhibitor TSA on reversing the pathological effects of 6-hydroxydopamine (6-OHDA) in the destruction of nigrostriatal dopaminergic neurons was investigated.

\section{Material and methods}

\section{Chemicals and kits}

6-OHDA hydrobromide, tubastatin A, and sodium hydrogen sulfide were obtained from Sigma Aldrich. RNA isolation kits, cDNA synthesis, and SYBR Green/ ROX master mix were from Qiagen. Primer sequences for PCR were obtained from Eurofins MWG (Operon). Protein carbonyl, lipid peroxidation, superoxide dismutase (SOD), catalase (CAT), and glutathione (GSH) assays kits were obtained from Cayman Chemicals, USA. The HDAC activity assay kit was obtained from Biomol, PA, USA. The HAT activity assay kit was acquired from Active Motif, Carlsbad, CA, USA. The Fluoro-Jade $C$ assay kit was obtained from Millipore Sigma, Burlington, MA, USA. GFAP monoclonal antibody and the corresponding anti-mouse secondary antibody were obtained from Santa Cruz Biotechnology, USA. ELISA kits for IL-1 $\beta$ (ER1094), IL-2 (ER0039), IL-17 (ER0035), IL-8 (ER1623), IL-6 (ER0042), TH (ER0534), GFAP (ER0229), $\alpha$-synuclein (ER0921), and TNF- $\alpha$ (ER1393) were obtained from Fine Biotech, China. All other chemical reagents used were analytical grade.

\section{PD rat model}

The experimental method was accepted by the Institutional Animal Care and Use Committee, Department of Rehabilitation Medicine, The Second Affiliated Hospital of Xi'an Medical University, Xi 'an, Shaanxi, China. All animal works were carried out strictly in accordance with institutional committee regulations. For the study, Wistar male rats weighing 160-200 g were used in the study. The animals were anesthetized with ketamine and xylazine (100: $25 \mathrm{mg} / \mathrm{kg}$, i.p.) and placed in a ste- 
reotaxic apparatus. Using the Hamilton syringe $0.46 \mathrm{~mm}$ diameter blunt needlepoint at a rate of $0.5 \mu \mathrm{l}$ per min, 6-OHDA (10 $\mu \mathrm{g}$ of 6-OHDA hydrobromide dissolved in $5 \mu \mathrm{l}$ germ-free saline comprising $0.02 \%$ L-ascorbic acid) was injected into the left striatum (coordinates from bregma: $\mathrm{AP},+1.0 \mathrm{~mm}$; $\mathrm{ML},+3.0 \mathrm{~mm}$; DV, $-4.5 \mathrm{~mm}$ ). Concurrently, sham-operated rats were injected with $5 \mu \mathrm{l}$ of saline containing $0.02 \% \mathrm{~L}$-ascorbic acid into the left striatum and used as controls. After the induction, the rats were maintained separately in cages and exposed to a $12: 12 \mathrm{~h}$ light-dark cycle with unrestricted access to tap water and food for 5 weeks. The induction of PD was confirmed by apomorphine-induced rotations as described earlier [27].

\section{Experimental group}

Wistar rats were separated into four experimental groups as follows: Set 1: Control (Saline treated); Set 2: PD induced; Set 3: PD + NaHS; Set 4: PD + tubastatin A (TSA). The doses and the administration protocol of NaHS [28] and TSA [29] were selected based on the results from prior publications. After the experimental period, the animals were killed; brain tissues were removed and saved at $-80^{\circ} \mathrm{C}$ until analysis. A portion was fixed in $10 \%$ phosphate-buffered formalin, processed and embedded in paraffin blocks. Further $5 \mu \mathrm{m}$ thick sections made from blocks were used for hematoxylin and eosin (H\&E) staining or unstained for immunofluorescence analysis. Additionally, to reveal the neurons undergoing degeneration, the fluorescein derivative stain Fluoro-Jade was used. Briefly, the paraffin sections were rehydrated and incubated in $0.06 \%$ potassium permanganate $\left(\mathrm{KMnO}_{4}\right)$ solution for 15 min, washed in distilled water, and transferred to a Fluoro-Jade solution for $30 \mathrm{~min}$ and washed and observed with a fluorescence microscope.

\section{Apomorphine-induced rotations test}

The imbalance in the nigrostriatal dopaminergic pathways or nigrostriatal bundle (NSB), between the right (lesioned) and left (unlesioned) brain hemispheres on apomorphine administration causes the changes in rotational behavior. The intraperitoneal injection of apomorphine $(1 \mathrm{mg} / \mathrm{kg}$ ) induced contralateral rotation (opposite to the lesioned right side) was monitored for $1 \mathrm{~h}$ and the number of rotations was recorded and compared between groups.

\section{Biochemical, cytokine, HDAC and HAT analysis}

At the end of drug treatments, the brain tissues were harvested, homogenized in radioimmunoprecipitation assay buffer (RIPA buffer) and centrifuged $(10000 \times \mathrm{g})$ for $20 \mathrm{~min}$ at $4^{\circ} \mathrm{C}$ for the extraction of protein. For the biochemical analysis, commercial kits from Cayman chemicals were used. For analysis of lipid peroxidation, protein carbonyl content, the antioxidant enzymes SOD, CAT, glutathione peroxidase (GPx) and reduced GSH, the assays were performed as per the manufacturer's protocol. The amount of nitric oxide in the brain tissue was quantified using Griess reagent. In order to determine the levels of cytokines in the striatum of control and experimental animals, brain tissue of rats was prepared and the cytokines were analyzed as per the manufacturer's guidelines. Further, using the commercial assay kits to analyze HDAC and HAT activity, the nuclear protein was extracted from the brain tissues and incubated with corresponding HDAC and HAT activity assay buffers with the substrate and the reaction was developed and the total activity was quantified using a fluorimeter as per the manufacturer's instructions.

\section{Analysis of dopamine and its metabolite}

The DA and DOPAC (3, 4-dihydroxyphenylacetic acid) content in the striatal area was determined by high performance liquid chromatography (HPLC). The brain tissue homogenates were prepared in $10 \% \mathrm{HClO}_{4}$ and centrifuged (10000×g) for $15 \mathrm{~min}$ at $4^{\circ} \mathrm{C}$ and the supernatant was used as the sample to inject $(20 \mu \mathrm{l})$ into the HPLC column. The Shimadzu L-ECD-6A electrochemical detector coupled Shim-Pak CLC- ODS column $(25 \mathrm{~cm}$ length) with the flow volume of $0.6 \mathrm{ml} / \mathrm{min}$ was used. The mobile phase $(\mathrm{pH} 3.0)$ was composed of $150 \mathrm{mM}$ monohydrated citric acid, $67 \mathrm{mM}$ sodium octyl sulfate, $2 \%$ tetrahydrofuran, and $4 \%$ acetonitrile in deionized water. The retention time of the analytes was determined using the standard monoamines and test samples were quantified by comparison with standards and the results are expressed as ng/g wet tissue.

\section{Confocal immunofluorescence staining}

The immunofluorescence staining was carried out according to the methods as earlier reported with slight modification [30]. The paraffin sections were hydrated using xylene and series of ethanol and distilled water. The brain sections were permeabilized with Triton X-100/PBS (0.3\%) for $10 \mathrm{~min}$ and blocked with BSA (10\%) in PBS for another $30 \mathrm{~min}$. After that, the sections were incubated with mouse monoclonal GFAP antibody (1:500, Sigma) for $2 \mathrm{~h}$ at $23^{\circ} \mathrm{C}$ and followed with appropriate goat anti-mouse secondary antibody incubation for $1 \mathrm{~h}$. After $1 \mathrm{~h}$ of incubation, the cells were washed three times with PBS and counterstained using DAPI and visualized under a Zeiss LSM 700 confocal microscope (magnification 20x). 
Table I. Oligonucleotides used in this study

\begin{tabular}{|c|c|c|c|c|}
\hline Gene & Primer & Sequence & Annealing & Accession number \\
\hline \multirow[t]{2}{*}{ HDAC2 } & $\mathrm{F}$ & CATGCGCCTGGTGTTCAAAT & 59 & NM_053447.1 \\
\hline & $\mathrm{R}$ & TCAGCGACATTCCTACGACC & & \\
\hline \multirow[t]{2}{*}{ HDAC3 } & $\mathrm{F}$ & ACACCCGATGAAACCCCATC & 59 & NM_053448.1 \\
\hline & $\mathrm{R}$ & TCAGAATGGAAGCGGCACAT & & \\
\hline \multirow[t]{2}{*}{ HDAC4 } & $\mathrm{F}$ & TGGAAGAGCTGCAGACAGTG & 57 & NM_053449.1 \\
\hline & $\mathrm{R}$ & TCAGCGAGCTGTCCAGTTTC & & \\
\hline \multirow[t]{2}{*}{ HDAC6 } & $\mathrm{F}$ & CCATGCCATCAAAGAGCAGC & 56 & KY009929.1 \\
\hline & $\mathrm{R}$ & TAGGTGCCTGCAAGAACTCG & & \\
\hline \multirow[t]{2}{*}{ GAPDH } & $\mathrm{F}$ & AGTGCCAGCCTCGTCTCATA & 58 & NM_017008.4 \\
\hline & $\mathrm{R}$ & GGTAACCAGGCGTCCGATAC & & \\
\hline
\end{tabular}

\section{Reverse transcription-PCR}

Total RNA was extracted from the brain tissue using TRIzol reagent. The brain tissue was homogenized in TRIzol and then maintained at $23^{\circ} \mathrm{C}$ for $5 \mathrm{~min}$. To this chloroform was added and tubes were mixed forcefully and were centrifuged $(12000 \times g)$ for $15 \mathrm{~min}$ at $4^{\circ} \mathrm{C}$. The colorless upper aqueous stage was moved to a new tube containing isopropanol and incubated for $10 \mathrm{~min}$ at $25^{\circ} \mathrm{C}$ followed by centrifugation (12000*g) for $10 \mathrm{~min}$ at $4^{\circ} \mathrm{C}$. The obtained RNA was washed with $70 \%$ ethanol and the concentrations were determined using a NanoDrop Spectrophotometer 2000C (Thermo Fisher Scientific, USA). An equal amount of RNA was transcribed to CDNA using the iScript cDNA synthesis kit. The real-time RT-PCR was done for specific genes using SYBR green/ ROX master mix and amplified using the Bio-Rad real-time PCR system and data were analyzed by comparing with control house keeping genes. The forward ( $F$ ) and reverse (R) primers used for the specific genes are given in Table I. The PCR reaction was carried out with the following conditions: $95^{\circ} \mathrm{C}$ for $10 \mathrm{~min} ; 32 \mathrm{cycles}$ at $95^{\circ} \mathrm{C}, 10 \mathrm{~s}$ and $60^{\circ} \mathrm{C}$, $1 \mathrm{~min}$; followed by 1 cycle at $95^{\circ} \mathrm{C}, 1 \mathrm{~min}, 55^{\circ} \mathrm{C}$, $30 \mathrm{~s}$ and $95^{\circ} \mathrm{C}, 30 \mathrm{~s}$ for the detachment curve. The fold increase in the gene transcript was calculated from the $C t$ values and the gene expression was determined by the comparative $C t$ method $(\Delta \Delta C t)$ with expression values of glyceraldehyde 3-phosphate dehydrogenase (GAPDH) as an endogenous control.

\section{Statistical analysis}

Statistical significance was evaluated with a one-way analysis of variance (ANOVA) followed by a post hoc (Bonferroni) test for multiple group comparison. For comparison of two groups, Stu- dent's $t$-test was used. Differences with a $p$-value less than 0.05 were considered statistically significant.

\section{Results}

To evaluate the role of the epigenetic mediated mechanism in the protective effect exerted by hydrogen sulfide, in the present study, rats were selected and unilateral lesions were made with 6-hydroxydopamine (6-OHDA) to induce PD. In the current investigation, the experimentally made lesions in the brain tissues showed obvious damage in the brain tissues histology. The photomicrographs of another group of rats exposed to sodium hydrosulfide (NaHS) and trichostatin A (TSA) demonstrated a reduction in the damage that may be a reversal of neurons' development (Figure $1 \mathrm{~A}$ ). Further, the results on the time course of the apomorphine-induced rotation study elicited $(p<0.001)$ a marked contralateral rotation toward the intact hemisphere from week 4 to 7 after injection of 6-OHDA into the left striatum compared to the control, in which no rotation was observed even after $60 \mathrm{~min}$. On the other hand, apomorphine-induced rotational behavior was significantly $(p<0.01)$ reduced in the NaHS and TSA administered group compared to the 6-OHDA-lesioned group (Figure $1 \mathrm{~B}$ ).

Further, to evaluate the cellular oxidant-antioxidant levels, the levels of lipid peroxidation (LPO), protein carbonyl (PCO), and nitric oxide (NO) were analyzed in the brain tissues of PD-induced rats and the results demonstrated increasing levels of LPO including PCO with elevated nitric oxide levels; while SOD, CAT and GSH were shown to have depleted levels. Conversely, PD rats administered with NaHS and TSA showed restored $(p<0.05)$ anti-oxidant levels with reduced oxidant levels (Figure 2). 
A
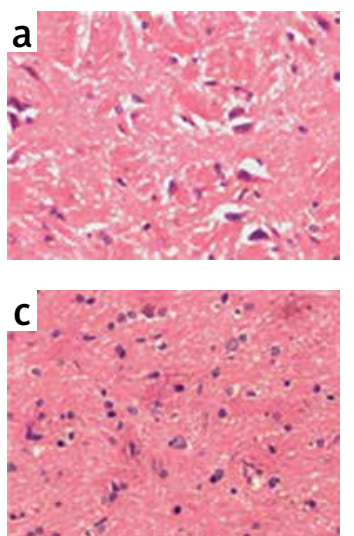

B
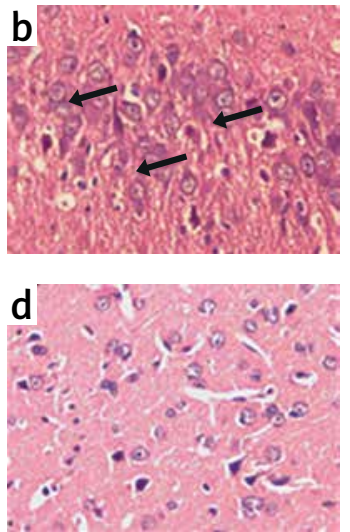

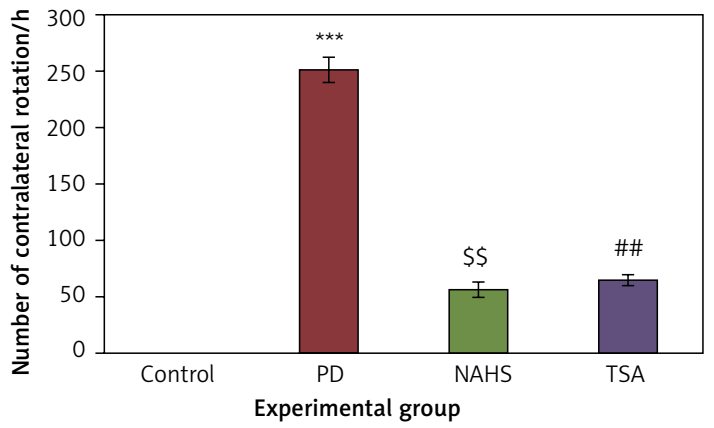

Figure 1. A - Histopathological analysis in the brain tissue of control and experimental groups of rats. Figure inseta, c \& d. Normal brain architecture of control (group 1), NAHS treated PD rats (group 3) and TSA treated PD rats (group 4). Brain tissue architecture of PD induced rats was quite well-differentiated from the surrounding tissue with richly cellular, nuclear changes and increased anaplasia was found (group 2); B - The apomorphine-induced contralateral rotations of control and experimental groups of rats

Values are expressed as mean $\pm S E(n=6)$. Statistical significance expressed as ${ }^{* * *} p<0.001$ compared to saline-treated controls, ${ }^{s S} p<0.01$ NAHS compared to PD rats; ${ }^{\# \#} p<0.01$ TSA compared to PD rats.
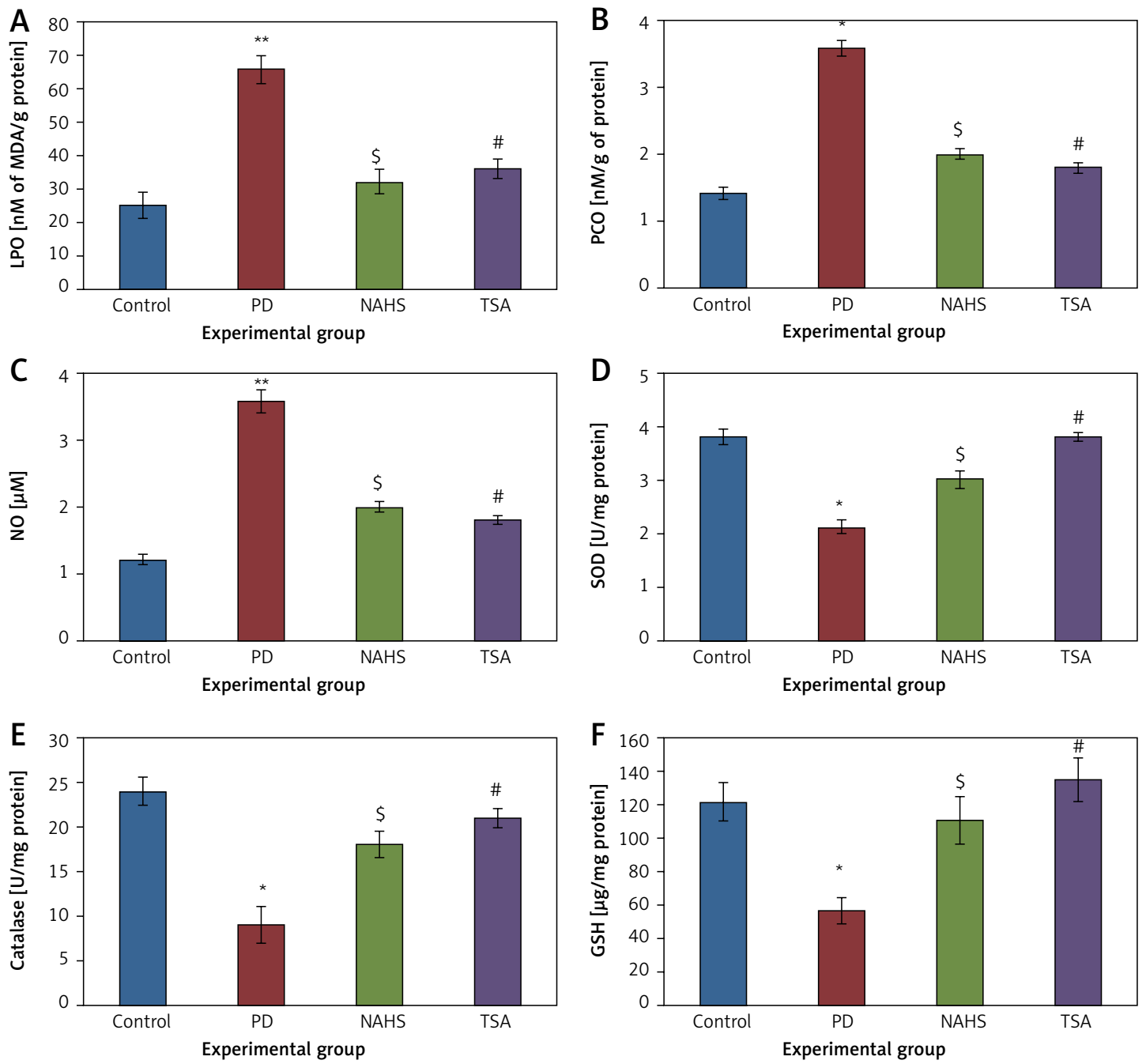

Figure 2. A-F - Represents the levels of LPO, PCO, NO, SOD, catalase, and glutathione in control and experimental group of rats. The units for LPO are represented as nmoles of MDA released/g of protein; PCO as nmol/mg protein. The enzyme activity unit for SOD and catalase is expressed as units/mg protein; GSH as $\mu g / \mathrm{mg}$ protein Values are expressed as mean $\pm S E(n=6)$. Statistical significance expressed as ${ }^{*} p<0.05,{ }^{* *} p<0.01$ compared to saline-treated controls, ${ }^{s} p<0.05$ NAHS compared to PD rats; ${ }^{*} p<0.05$ TSA compared to PD rats. 
Moreover, Figure 3 shows the Fluoro-Jade staining which demonstrated the neuron pattern. In the present study, in the 6-OHDA-lesioned group, an increased number of Fluoro-Jade stained degenerating neurons in the striatum that appears in bright green fluorescence compared to the control. The observed fluorescence was diminished in the NaHS and TSA treated group, which indicates that less neuronal degeneration was found in PD-developed animals. The histogram represents the relative fluorescence observed between groups measured in 3 to 5 fields and quantified by the Image J software. The presynaptic neuronal protein $\alpha$-synuclein, a Lewy body indicator protein, was found to be attenuated $(p<0.01)$ in the $\mathrm{NaHS}$ and TSA group, which was increased 3 -fold in the PD group, showing that the $\mathrm{H}_{2} \mathrm{~S}$ or HDAC inhibition exerts protection (Figure 3 ).

Further, the protective effect of NaHS and TSA on the levels of neurochemical was evaluated in PD induced rats, as depicted in Figure 4. The levels of dopamine and DOPAC in the lesioned side of the 6-OHDA group showed significantly $(p<0.01)$ reduced levels compared to the control. The levels of decreased dopamine (DA) and DOPAC contents were dramatically raised to levels $(p<0.05)$ equivalent to the control in both NaHS and TSA administered groups (Figure 4).

From the above results, the neurochemical abnormality was observed in PD animals that might be due to the defective enzymatic pathway that leads to the metabolite synthesis. Hence, the level
A
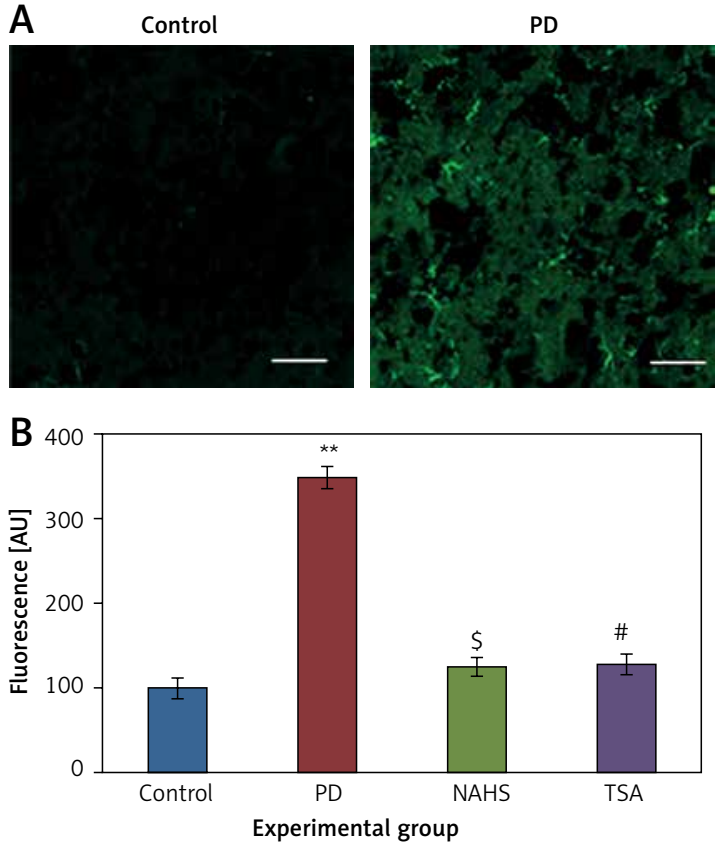
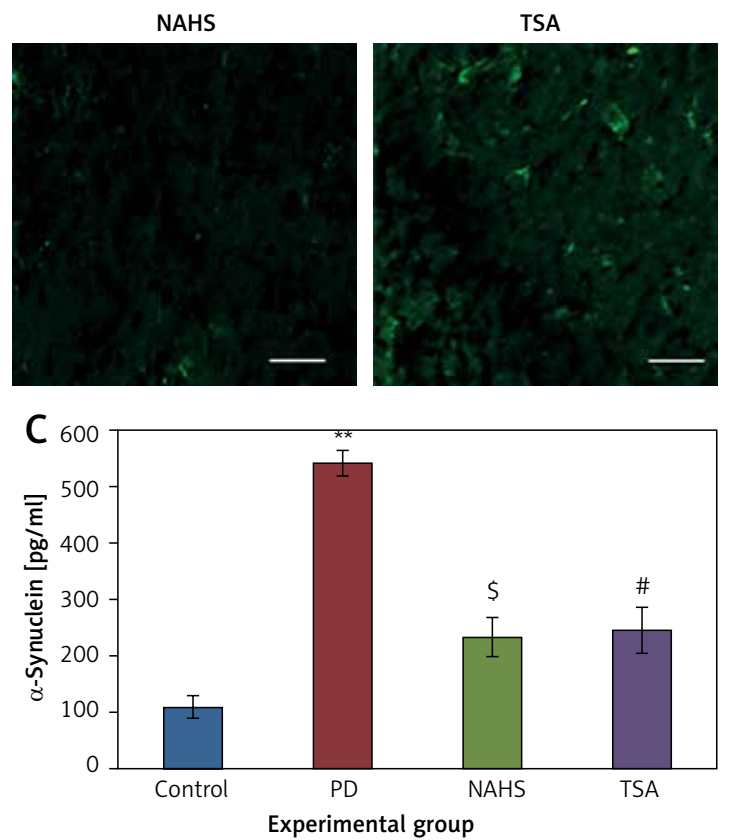

Figure 3. A - Representative confocal microscopic image of Fluoro-Jade staining of control and experimental group of rats' brain tissue. B - The fluorescence intensities were quantified and represented as arbitrary units (magnification 20x). C - The level of $\alpha$-synuclein measured using ELISA is shown

Statistical significance expressed as ${ }^{*} p<0.05,{ }^{* *} p<0.01$ compared to saline-treated controls, ${ }^{5} p<0.05$ NAHS compared to PD rats; $" p<0.05$ TSA compared to PD rats.

\section{A}

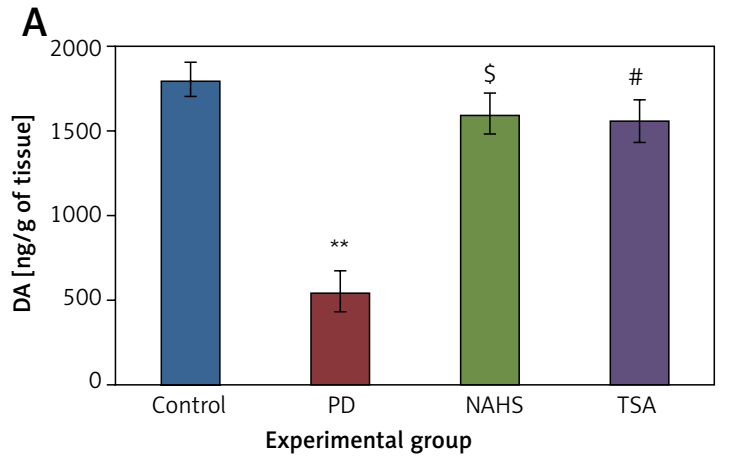

B

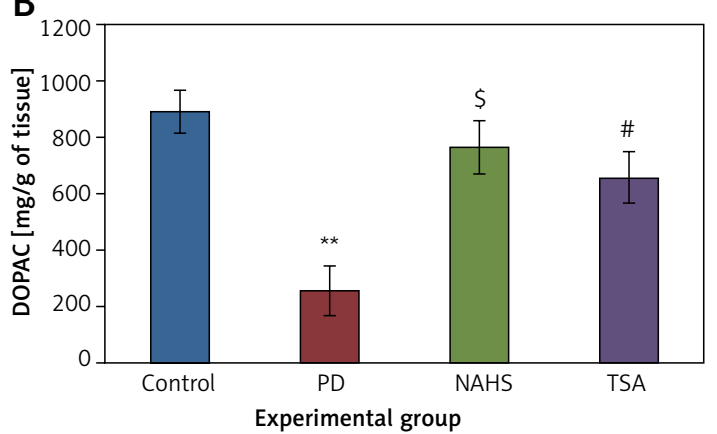

Figure 4. A, B - The level of dopamine (DA) and DOPAC contents in control and experimental group of rats. The amount of DA and DOPAC is expressed as $\mathrm{ng} / \mathrm{g}$ tissue

Values are expressed as mean $\pm S E(n=6)$. Statistical significance expressed as ${ }^{*} p<0.05,{ }^{* *} p<0.01$ compared to saline-treated controls, ${ }^{s} p<0.05$ NAHS compared to PD rats; ${ }^{*} p<0.05$ TSA compared to PD rats. 
A
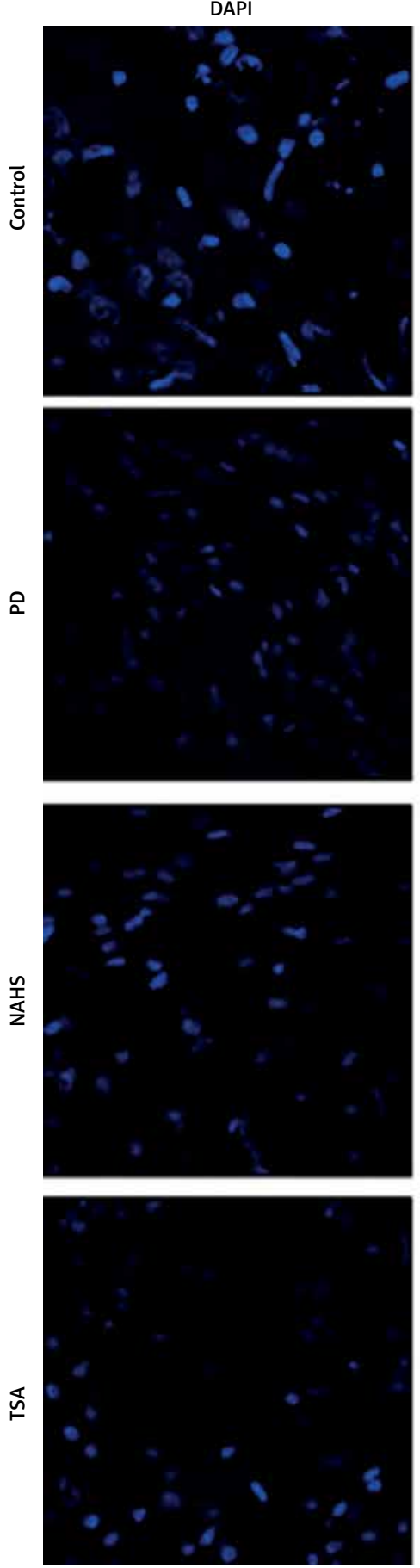

GFAP
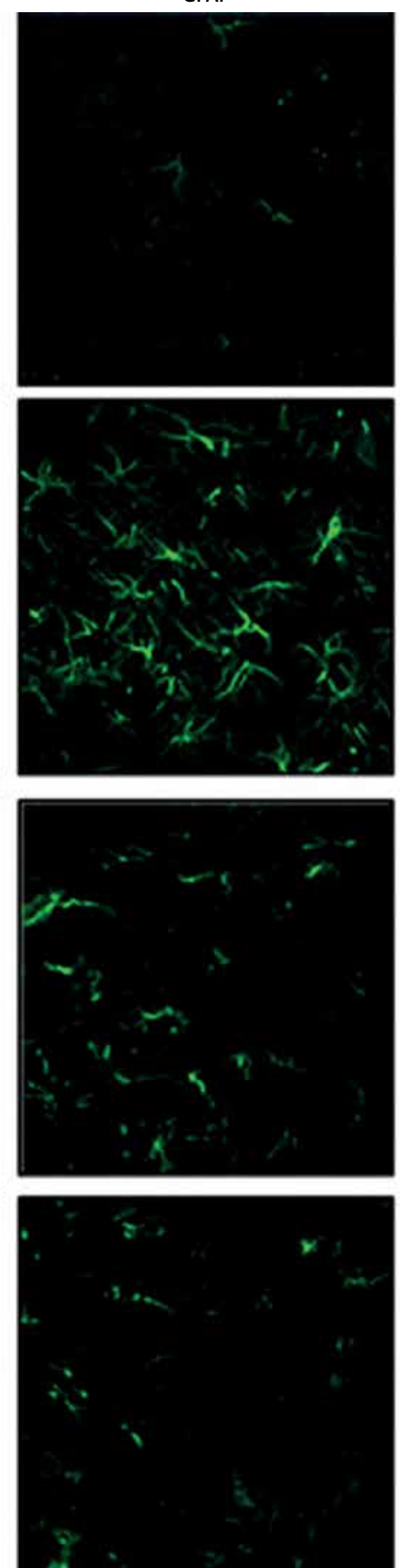

Merge
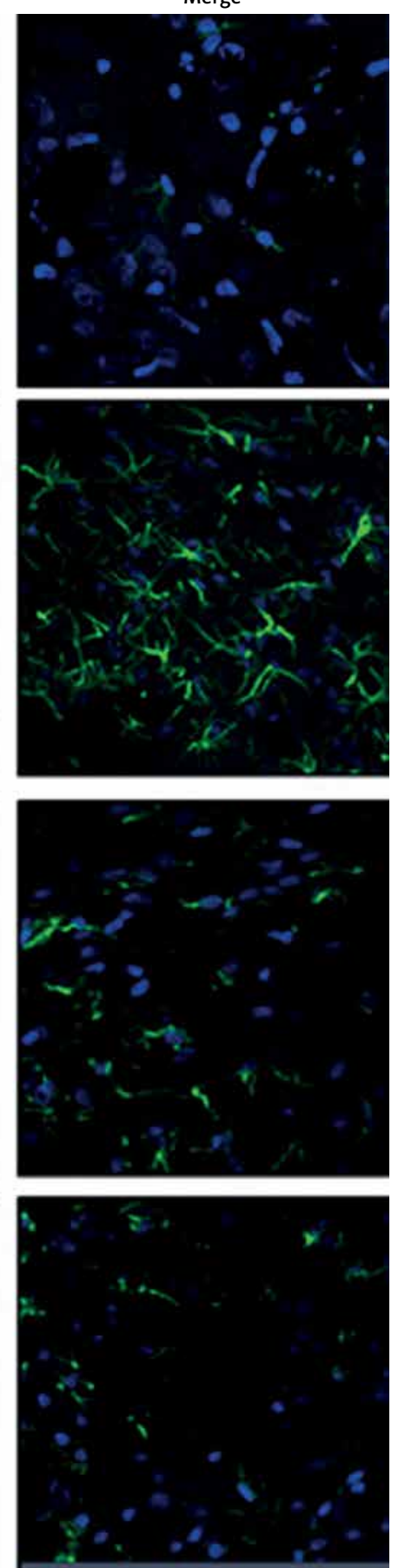

Figure 5. A - Immunofluorescence and densitometric analysis of GFAP protein. The details of the staining protocol are given in the methodology section. The fluorescence intensities have been quantified and compared between groups

of TH was analyzed using a commercial ELISA kit and the results demonstrate that the levels of $\mathrm{TH}$ were significantly $(p<0.01)$ reduced in PD induced animals. At the same time, the immunofluorescence analysis revealed that there was an increase in GFAP positive cells compared to the control. On the other hand, rats under NaHS and TSA exposure presented improved $(p<0.05)$ TH levels with reduced immunoreactivity of GFAP as fluorescence, suggesting that physiological functions related to movement control and astrocyte-neuron interactions are efficiently protected in NaHS and TSA treatment (Figure 5).

Further, to substantiate the role of $\mathrm{H}_{2} \mathrm{~S}$ in the modulation of histone deacetylases (HDACs), the mRNA levels of HDACs were identified in the pres- 
B
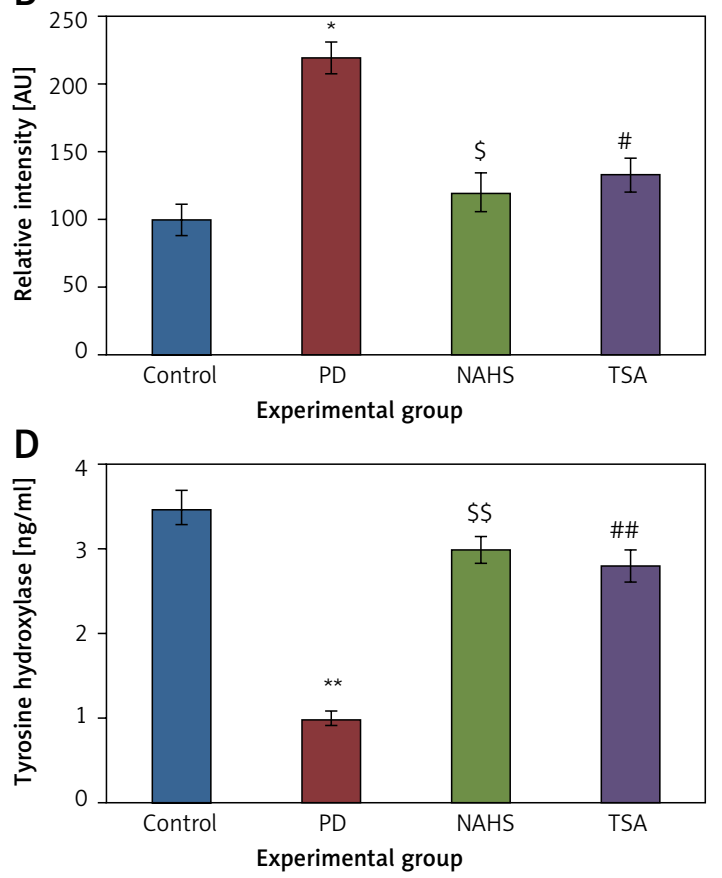

ent study and the results of the expression levels of HDACs explained by real-time quantitative PCR in relation to the control gene are shown in Figure 6. The results show a profound upsurge $(p<0.01)$ in the mRNA expression of HDAC-2 (4-fold), HDAC-3 (5.2-fold), HDAC-4 (2-fold), HDAC-6 (3-fold) with total HDAC and reduced HAT activity in PD rats compared to the control. However, the enhanced levels of these HDAC genes were found reduced in NaHS and TSA treatment, indicating that the drug has initiated the restoring mechanism to maintain the normal functioning (Figure 6).

Conversely, an additional experiment on illuminating the cytokine levels was carried out and the results are presented in Figure 7 . In the present study, rats with PD induced using 6-OHDA-lesions demonstrated a significant increase in the levels of cytokines such as TNF- $\alpha(p<0.01), \mathrm{IL}-2(p<$ $0.05)$, IL-17A ( $p<0.01)$, IL-6 ( $p<0.001)$, and IL-1 $\beta$ $(p<0.05)$ compared to the control. These inflammatory cytokines were diminished in the $\mathrm{NaHS}$ and TSA treatment, indicating that the signaling of PD progression is reduced on HDAC inhibition (Figure 7). Thus the results of the current investigation demonstrate that the protective role of $\mathrm{H}_{2} \mathrm{~S}$ may be mediated via HDAC inhibition, which can contribute to the development of novel treatment strategies in PD.

\section{Discussion}

Apoptosis or literally cell death is due to the response of the system to eliminate the injured cells [31]. In PD, the degeneration of DN is due to oxidative stress-induced apoptosis [32]. The induction

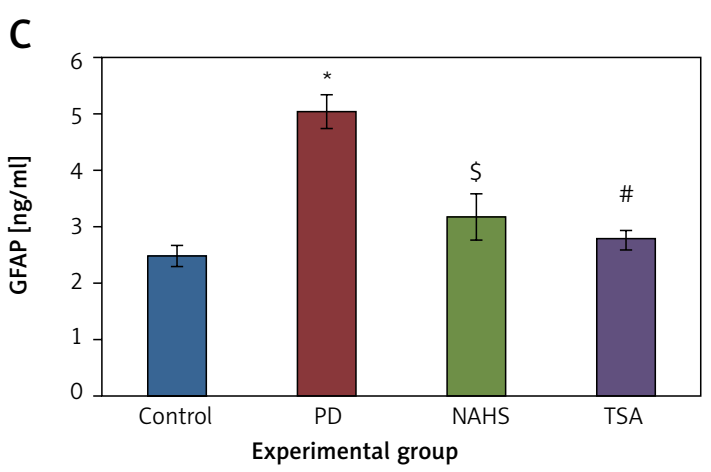

Figure 5. Cont. B - Immunofluorescence and densitometric analysis of GFAP protein. The details of the staining protocol are given in the methodology section. The fluorescence intensities have been quantified and compared between groups. C, D Levels of TH and GFAP levels measured using ELISA in control and experimental group of rats

Statistical significance expressed as ${ }^{*} p<0.05,{ }^{* *} p<0.01$ compared to saline-treated controls, ${ }^{s} p<0.05,{ }^{S S} p<0.01$ NAHS compared to PD rats; $" p<0.05$, ${ }^{\#} p<0.01$ TSA compared to $P D$ rats.

of cell death in the neurons of rats induced with 6-OHDA is due to the generation of ROS by autoxidation in the cytosol of the brain. The sustained effect of 6-OHDA is evident in the major contribution of free radicals and a simultaneous increase in lipid peroxidation due to LPO (Figure 2 B) in the striatum of the rats lesioned with 6-OHDA in PD-developed rats. The free radicals that are generated in PD mice have been shown to be dramatically reduced when treated with NHS and TSA. The reduction in the free radicals is made possible by the increase in oxidative enzymes such as SOD, CAT and GSH (Figures 2 D-F of NAHS and TSA), which serve as endogenous antioxidant defenses in the cells. The increase in these oxidant enzymes in the cells could also be due to the treatment of these cells with drugs, and in this case, it is with TSA. The effect of TSA would be to neutralize the inflicted damage of lipid peroxidation and protein nitration which is found in the brain tissues. Hydrogen peroxide tends to get eliminated by the enzymatic action of CAT and lipid peroxides along with free radicals would be removed by GSH in association with NADPH [33]. Hence the antioxidative mechanism of TSA in fighting the 6-OHDA-induced oxidative stress in PD animals can be better explained.

Lewy bodies are intracellular protein aggregates containing $\alpha$-synuclein as their major component. They are present in the pre-synaptic terminals and are first hand evidence of the neurodegeneration in PD $[18,34]$. The aggregation and accumulation of $\alpha$-synuclein could be a great challenge to the cellular machinery as the chap- 
A

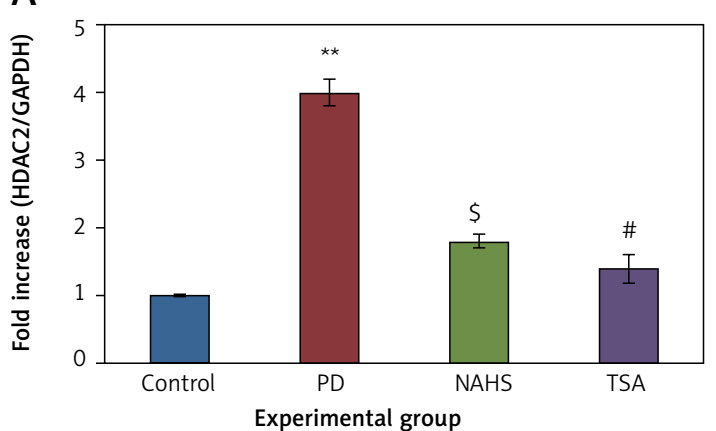

C
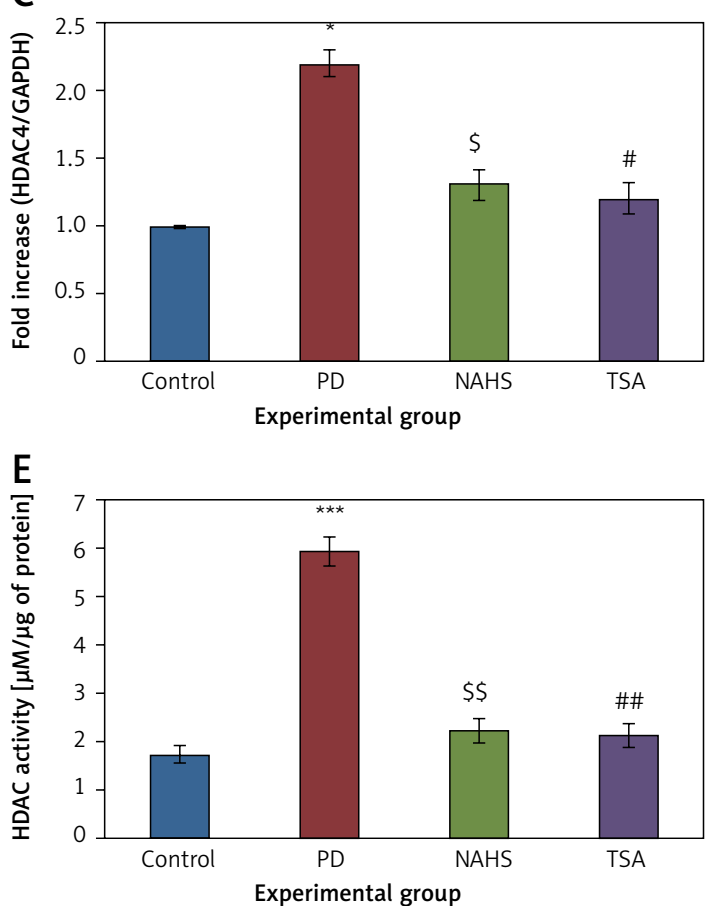

B

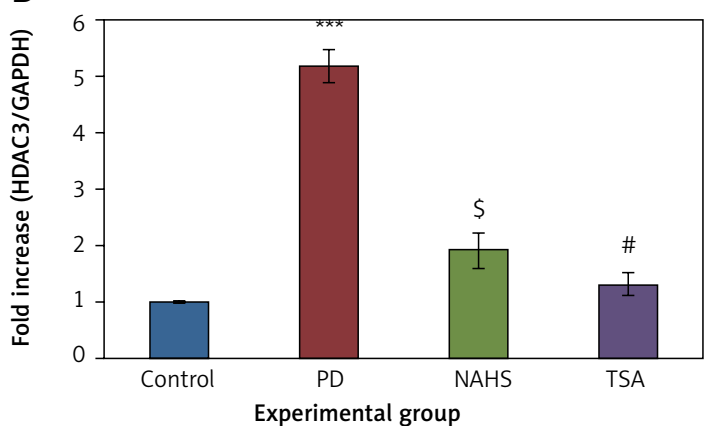

D

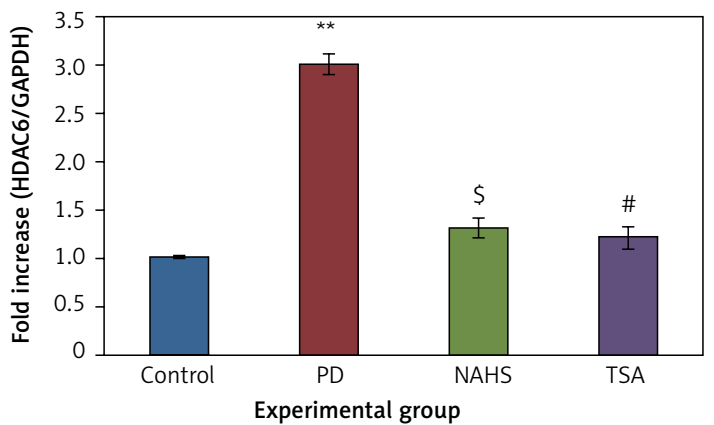

$\mathrm{F}$

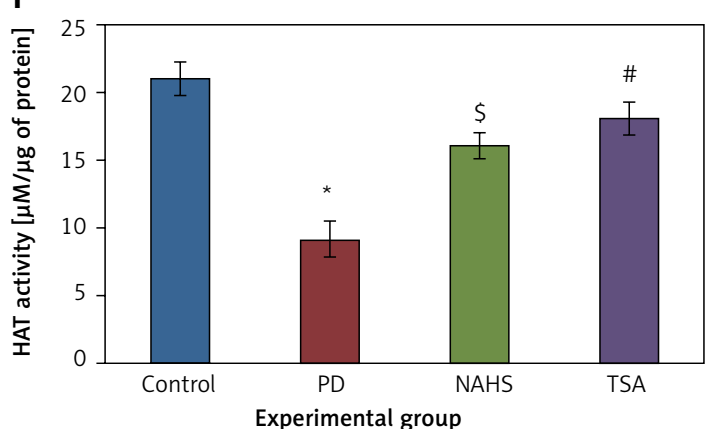

Figure 6. A-D - qRT-PCR mRNA expression analysis of HDAC-2, -3, -4, -6 respectively in control and experimental groups of cells. The fold increase of gene expression is compared with the housekeeping gene GAPDH. E, F- HDAC and HAT activity

Statistical significance expressed as ${ }^{*} p<0.05,{ }^{* *} p<0.01$, ${ }^{* * *} p<0.001$ compared to saline-treated controls, ${ }^{s} p<0.05,{ }^{s \$} p<0.01$ NAHS compared to PD rats; ${ }^{*} p<0.05,{ }^{\# \#} p<0.01$ TSA compared to PD rats.

erones [35], in PD-induced animals, would be ineffective in handling the overwhelmed population of aggregated proteins at the DA neurons. This tilts the cellular balance between protein synthesis and degradation. NAHS and TSA treatment in cells decreases the accumulation of aggregated $\alpha$-synuclein in 6-OHDA-treated cells, as can be seen in Figure 3, and hence decreases the overall toxicity of the cells due to its accumulation. According to Ximenes et al., the decrease in DA in the substantia nigra is due to the degeneration of neurons and also due to 'innervations' in the striatum caused by dopaminergic cell bodies. We showed in Figure 4 that the main symptom of PD is a defect in motor neurons and that is due to the loss of dopamine depletion in the neurons. This effect obtained in cells treated with 6-OHDA is completely reversed when these cells are treated with NHS or TSA to the control levels (Figure $4 \mathrm{~A}$ ). The neuroprotective effects of TSA are discerned from the reversal of DA and DOPAC to near-control levels when these cells are treated with NHS and TSA. Also, the levels of DOPAC-DA metabolite were restored to control levels with the treatment of cells with NHS or with TSA (Figure $4 \mathrm{~B}$ ).

In the first line of attack of 6-OHDA-induced PD, dopaminergic neurons are affected and the glial reactions that occur in this experimental model would next affect the astrocytes and it is also involved in the pathophysiology of PD. Astrocytes that are affected are enlarged and have a prominent expression of the glial fibrillary acidic protein (GFAP). We have shown in Figure $5 \mathrm{C}$ that induction of rats with 6-OHDA would increase 
A

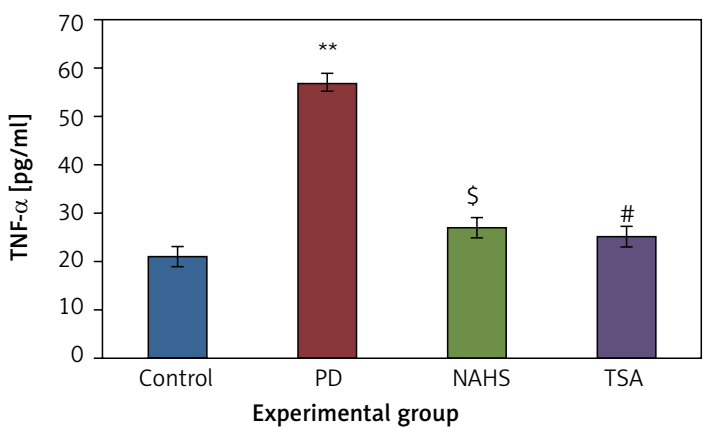

C

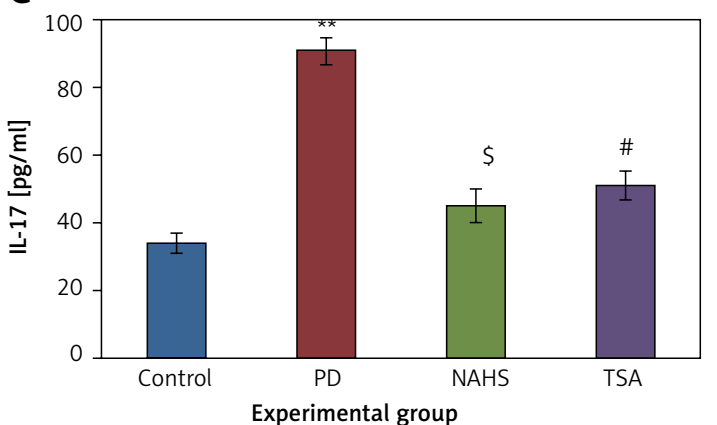

E

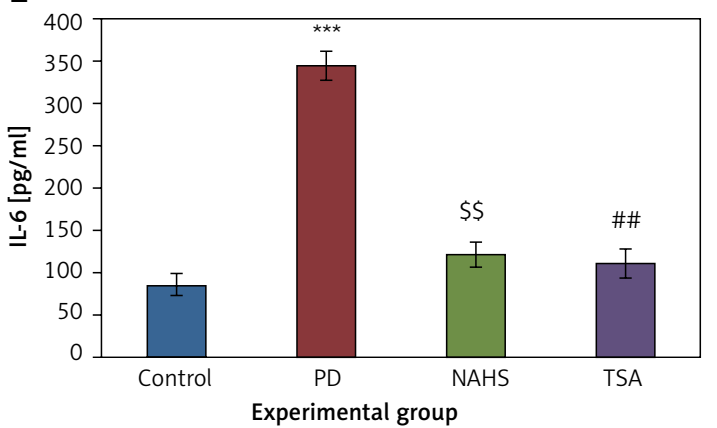

B

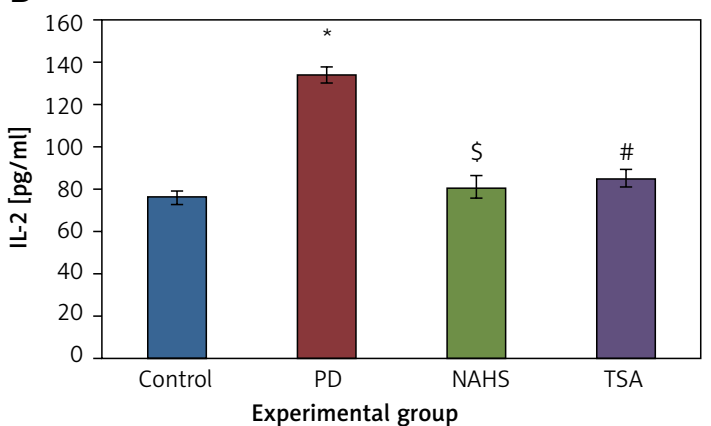

D

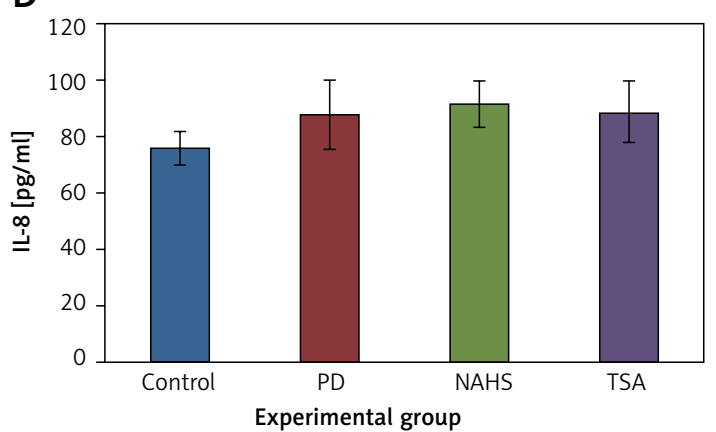

F

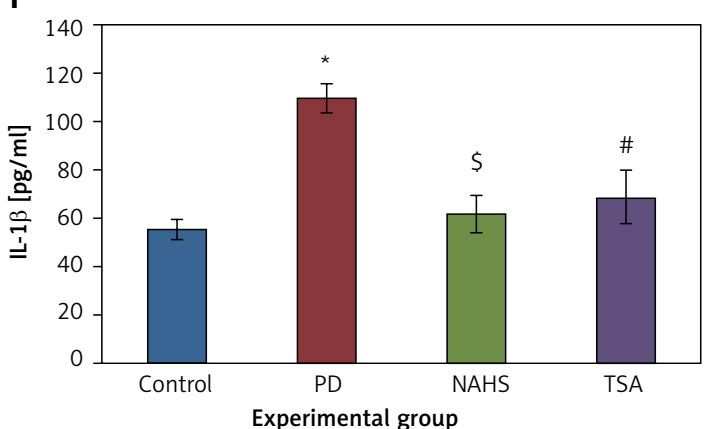

Figure 7. A-F - Cytokine expression analysis of TNF- $\alpha$, IL-2, IL-17, IL-8, IL-6, and IL-1 $\beta$, and presynaptic neuronal protein, $\alpha$-synuclein respectively in control and experimental groups of rats. The details of the experiment are given in the methodology section

Statistical significance expressed as ${ }^{*} p<0.05,{ }^{* *} p<0.01$, ${ }^{* * *} p<0.001$ compared to saline-treated controls, ${ }^{s} p<0.05,{ }^{s s} p<0.01$ NAHS compared to PD rats; $p<0.05,{ }^{\# \#} p<0.01$ TSA compared to PD rats.

the expression of GFAP in astrocytes and that the treatment with TSA has decreased it. This indicates that the behavioral deficits such as akinesia, bradykinesia, and defects in the use of paws and forelimbs would be rectified with the therapeutic action of TSA. Similarly, the dopaminergic neurodegeneration evaluated by levels of tyrosine hydroxylase $(\mathrm{TH})$, the limiting enzyme for DA synthesis, in immunohistochemistry results shows that PD rats do show repeated dopaminergic toxic insults with reduced levels of $\mathrm{TH}$ and it is increased in intensity as ELISA results show with TSA actions (Figure 5 D).

The pathophysiology of PD is best explained by the neuroinflammation that is caused due to increase in 6-OHDA induction. Activated microglia secrete pro-inflammatory cytokines such as
TNF- $\alpha$, IL-1 $\beta$, and IL-6 due to 6-OHDA treatment in the cells [36] and contribute to the impairment of neurons and subsequent inflammation and neurodegeneration [37]. The increase in these cytokines in the dopaminergic region acts as a compensatory response and a 'trigger' [38] to neurodegeneration. The blood-brain barrier compromised in PD $[39,40]$ could lead to infiltration of $T$ and $B$ cells and that would generate IL- $1 \beta, \mathrm{IL}-6$, and TNF- $\alpha$ to stimulate inflammatory reactions in neurons [41]. The toxic effects of these cytokines contribute to the pathophysiology of PD [42-44]. These are the clinical evidence contributing to the inflammatory response in PD [45].

We have also found IL-17 to be increased in PD. But we are not excited to report that a 'direct correlation' exists between induction of PD in rats 
and IL-17 generation. The trends are reversed with the action of TSA on PD-induced cells (Figure 6) wherein the rise of NO due to oxidative stress is curtailed and hence decreases the expression of TNF- $\alpha$, IL- $1 \beta$, and IL- 6 to control levels.

In conclusion, in the present study, it has been shown that TSA has protective action against Parkinson's properties and symptoms in the 6-OHDA-induced PD in a rat model. This is significant assuming its actions against neuroinflammation by fighting against the pro-inflammatory cytokines by increasing the oxidant enzymes and also simultaneously inhibiting HDAC6 from transcribing them. Also, the toxic effects arising from protein aggregates of $\alpha$-synuclein are reduced. Further contributions from TSA include resuscitation of DA and DOPAC in inflamed neurons. Hence, we propose to use TSA as a primary candidate in PD clinical trials to investigate further the therapeutic mechanism in the treatment of PD.

\section{Acknowledgments}

Yang Sun and Dai Li contribute to this work equally.

\section{Conflict of interest}

The authors declare no conflict of interest.

\section{References}

1. Moore DJ, West AB, Dawson VL, Dawson TM. Molecular pathophysiology of Parkinson's disease. Annu Rev Neurosci 2005; 28: 57-87.

2. Essawy SS, Tawfik MK, Korayem HE. Effects of adenosine receptor antagonists in MPTP mouse model of Parkinson's disease: mitochondrial DNA integrity. Arch Med Sci 2017; 13: 659-69.

3. Parkinson J. An essay on the shaking palsy. Sherwood, Neely, Jones, London, 1817. Reprinted in: J Neuropsychiatry Clin Neurosci 2002; 14: 223-36.

4. Yoo MS, Chun HS, Son JJ, et al. Oxidative stress regulated genes in nigral dopaminergic neuronal cells: correlation with the known pathology in Parkinson's disease. Brain Res Mol Brain Res 2003; 110: 76-84.

5. Jiang C, Wan X, Jankovic J, et al. Dopaminergic properties and experimental anti-parkinsonian effects of IPX750 in rodent models of Parkinson disease. Clin Neuropharmacol 2004; 27: 63-73.

6. Lee YM, Park SH, Shin DI, et al. Oxidative modification of peroxiredoxin is associated with drug-induced apoptotic signaling in experimental models of Parkinson disease. J Biol Chem 2008; 283: 9986-98.

7. Luquin MR. Experimental models, of Parkinson disease. Rev Neurol 2000; 31: 60-6.

8. Szabo C. Hydrogen sulphide and its therapeutic potential. Nat Rev Drug Discov 2007; 6: 917-35.

9. Kapoor A, Thiemermann C. Hydrogen sulfide, neurogenic inflammation, and cardioprotection: a tale of rotten eggs and vanilloid receptors. Crit Care Med 2010; 38: 728-30.

10. Lee SW, Hu YS, Hu LF, et al. Hydrogen sulphide regulates calcium homeostasis in microglial cells. Glia 2006; 54: 116-24.
11. Kimura H. Hydrogen sulfide induces cyclic AMP and modulates the NMDA receptor. Biochem Biophys Res Commun 2000; 267: 129-33.

12. Nagai $Y$, Tsugane M, Oka J, Kimura $H$. Hydrogen sulfide induces calcium waves in astrocytes. FASEB J 2004; 18 : 557-9.

13. Kimura Y, Goto Y, Kimura H. Hydrogen sulfide increases glutathione production and suppresses oxidative stress in mitochondria. Antioxid Redox Signal 2010; 12: 1-13.

14. Kimura $Y$, Kimura $H$. Hydrogen sulfide protects neurons from oxidative stress. FASEB J 2004; 18: 1165-7.

15. Zhou CF, Tang XQ. Hydrogen sulfide and nervous system regulation. Chin Med J 2011; 124: 3576-82.

16. Giuliani D, Ottani A, Zaffe D, et al. Hydrogen sulfide slows down progression of experimental Alzheimer's disease by targeting multiple pathophysiological mechanisms. Neurobiol Learn Mem 2013; 104: 82-91.

17. Dexter DT, Jenner P. Parkinson disease: from pathology to molecular disease mechanisms. Free Radic Biol Med 2013; 62: 132-44

18. Spillantini MG, Schmidt ML, Lee VM, Trojanowski JQ, Jakes R, Goedert M. Alpha-synuclein in Lewy bodies. Nature 1997; 388: 839-40.

19. Hirsch EC, Breidert T, Rousselet E, Hunot S, Hartmann A, Michel PP. The role of glial reaction and inflammation in Parkinson's disease. Ann N Y Acad Sci 2003; 991: 214-28.

20. Dietz KC Casaccia P. HDAC inhibitors and neurodegeneration: at the edge between protection and damage. Pharmacol Res 2010; 62: 11-7.

21. Saha RN, Pahan K. HATs and HDACs in neurodegeneration: a tale of disconcerted acetylation homeostasis. Cell Death Differ 2006; 13: 539-50.

22. Rouaux C, Jokic N, Mbebi C, Boutillier S, Loeffler JP, Boutillier AL. Critical loss of CBP/p300 histone acetylase activity by caspase- 6 during neurodegeneration. EMBO J 2003; 22: 6537-49.

23. Harrison IF, Crum WR, Vernon AC, Dexter DT. Neurorestoration induced by the HDAC inhibitor sodium valproate in the lactacystin model of Parkinson's is associated with histone acetylation and up-regulation of neurotrophic factors. Br J Pharmacol 2015; 172: 4200-15.

24. Monti B, Polazzi E, Batti L, Crochemore C, Virgili M, Contestabile A. Alpha-synuclein protects cerebellar granule neurons against 6-hydroxydopamine-induced death. J Neurochem 2007; 103: 518-30.

25. Monti B, Polazzi E, Contestabile A. Biochemical, molecular and epigenetic mechanisms of valproic acid neuroprotection. Curr Mol Pharmacol 2009; 2: 95-109.

26. Rane P, Shields J, Heffernan M, Guo Y, Akbarian S, King JA. The histone deacetylase inhibitor, sodium butyrate, alleviates cognitive deficits in pre-motor stage PD. Neuropharmacology 2012; 62: 2409-12.

27. Hu LF, Lu M, Tiong CX, Dawe GS, Hu G, Bian JS. Neuroprotective effects of hydrogen sulfide on Parkinson's disease rat models. Aging Cell 2010; 9: 135-46.

28. Sarukhani M, Haghdoost-Yazdi H, Sarbazi Golezari A, Babayan-Tazehkand A, Dargahi T, Rastgoo N. Evaluation of the antiparkinsonism and neuroprotective effects of hydrogen sulfide in acute 6-hydroxydopamine-induced animal model of Parkinson's disease: behavioral, histological and biochemical studies. Neurol Res 2018; 40: 523-31.

29. Jian W, Wei X, Chen L, et al. Inhibition of HDAC6 increases acetylation of peroxiredoxin $1 / 2$ and ameliorates 6-OHDA induced dopaminergic injury. Neurosci Lett 2017; 658: 114-20.

30. Azeez IA, Olopade F, Laperchia C, et al. Regional myelin and axon damage and neuroinflammation in the adult 
mouse brain after long-term postnatal vanadium exposure. J Neuropathol Exp Neurol 2016; 75: 843-54.

31. Pandurangan AK, Ismail S, Mohd Esa N, Munusamy MA Inositol-6 phosphate inhibits the mTOR pathway and induces autophagy-mediated death in HT-29 colon cancer cells. Arch Med Sci 2018; 14: 1281-8.

32. Bredesen DE. Neural apoptosis. Ann Neurol 1995; 38 839-51.

33. Vujotić L, Matić S, Borković-Mitić S, et al. Association between oxidative stress biomarkers and concentrations of some metal ions in the blood of patients with brain tumors and hydrocephalus. Arch Med Sci 2019; DOI: https://doi.org/10.5114/aoms.2019.87409.

34. Wakabayashi K, Matsumoto K, Takayama K, Yoshimoto $M$, Takahashi H. NACP, a presynaptic protein, immunoreactivity in Lewy bodies in Parkinson's disease. Neurosci Lett 1997; 239: 45-8.

35. Burre J, Sharma M, Sudhof TC. Alpha-Synuclein assembles into higher-order multimers upon membrane binding to promote SNARE complex formation. Proc Nat Acad Sci USA 2014; 111: E4274-83.

36. Pessoa Rocha N, Reis HJ, Vanden Berghe P, Cirillo C. Depression and cognitive impairment in Parkinson's disease: a role for inflammation and immunomodulation? Neuroimmunomodulation 2014; 21: 88-94.

37. Chen YM. Liu SP, Lin HL, et al. Irisflorentin improves alpha-synuclein accumulation and attenuates 6-OHDA-induced dopaminergic neuron degeneration, implication for Parkinson's disease therapy. Biomedicine 2015; 5: 4

38. Mogi M, Harada M, Narabayashi H, Inagaki H, Minami M, Nagatsu T. Interleukin (IL)-1 beta, IL-2, IL-4, IL-6 and transforming growth factor-alpha levels are elevated in ventricular cerebrospinal fluid in juvenile parkinsonism and Parkinson's disease. Neurosci Lett 1996; 211: 13-6.

39. Hirsch EC, Hunot S. Neuroinflammation in Parkinson's disease: a target for neuroprotection? Lancet Neurol 2009; 8: 382-97.

40. Kortekaas R, Leenders KL, van Oostrom JC, et al. Bloodbrain barrier dysfunction in parkinsonian midbrain in vivo. Ann Neurol 2005; 57: 176-9.

41. Wang Q, Liu Y, Zhou J. Neuroinflammation in Parkinson's disease and its potential as therapeutic target. Transl Neurodegener 2015; 4: 19.

42. Allan SM, Tyrrell PJ, Rothwell NJ. Interleukin-1 and neuronal injury. Nat Rev Immunol 2005; 5: 629-40.

43. Conroy SM, Nguyen V, Quina LA, et al. Interleukin-6 produces neuronal loss in developing cerebellar granule neuron cultures. J Neuroimmunol 2004; 155: 43-54.

44. Venters HD, Dantzer R, Kelley KW. Tumor necrosis factor-alpha induces neuronal death by silencing survival signals generated by the type I insulin-like growth factor receptor. Ann N Y Acad Sci 2000; 917: 210-20.

45. Xiao $M$. The role of proinflammatory cytokine interleukin-18 in radiation injury. Health Phys 2016; 111: 212-7. 